\title{
ENDODONTICS
}

\author{
The American Association of Endodontists
}

Victor H. Dietz, Editor

\section{BACTERIOLOGIC RESULTS FROM 4,000 ROOT CANAL CULTURES}

K. C. Winkler, M.D., and J. van Amerongen, Tandarts, Utrecht, Netherlands

INTRODUCTION

THE root canal culture is, in the first place, a test for sterility. Consepracticed. Aseptic technique is, nevertheless, requisite to endodontic treatment. If only as a check on aseptic technique, root canal culture is of important educational value.

The results of culturing depend partly on the medium used for primary culture. ${ }^{7,11,14,16}$ In a previous publication, ${ }^{1}$ we compared various media. Brain-heart infusion with 0.05 per cent agar in screw-capped bottles proved quite satisfactory and permitted the growth of most common aerobic and anaerobic organisms.

Whether or not the root eanal culture gives a true picture of the bacteriologic situation in the canal has been much discussed. It has been claimed, on the basis of theoretical considerations, that a negative culture from a positive canal would be a regular occurrence. Many authors, therefore, require two consecutive negative cultures before they will accept sterility of the canal.5, 8, 15 Van Amerongen ${ }^{2}$ showed, however, that with a suitable technique the probability for a positive culture after one or after two negative cultures is equal and low (2 to 3 per cent for experienced operators ; 8 to 9 per cent for students). The probability that the canal is sterile is not higher after two negative cultures than after one negative culture. Furthermore, large masses of dentine filed from the wall of a well-prepared canal generally were sterile if the normal paper-point culture had been sterile, giving further proof that negative cultures from root canals are representative in practice. The number of false positives is fairly high, of course, but it decreases with experience in aseptic techniques. ${ }^{2,9}$

From the Bacteriological Department, Laboratory of Hygiene, and the Department of Conservative Dentistry, University of Utrecht. 
A high percentage of infected canals become sterile by mechanical prepal. ration of the root canal. 5.17 In our experience, this percentage was 64 per. cent. $^{3}$ This phenomenon stresses the view that what yon take out of the canal is at least as important as what you put into it. In this connection, a contparison was made of the results obtained with chlorophenol-camphor-menthol and polyantibiotics. ${ }^{4}$ The results showed that although the number of applicattions necessary to obtain sterility was slightly smaller in the group treated with polyantibioties, the mean number of sessions was equal in both groups.

Regular culturing from root canals, starting with an initial culture during the first session, reduces the number of sessions necessary for treatment. The culture provides an invaluable check on the careful aseptic technique which is required for good root canal treatment. Without this regular check (that is, with less careful technique), the number of infected canals becoming sterile by preparation alone would be much less than 64 per cent. This leads to the remarkable paradox ${ }^{2}$ that root canal cultures are important for those who do not practice them and much less so for those who do. That a sound knowledge of the bacterial flora in intected canals is of importance in cases where focal infection is considered needs no comment.

Having advocated the practice of root canal cultures, we wish to report on the bacteriologic results of more than 4,000 cultures from more than 1,000 canals.

\section{MATERIALS AND METHODS}

Endodontic Techniques.-These have been described before. ${ }^{1}$ After extensive mechanical cleansing of the exterior of the tooth, the rubber-dam was applied (when necessary, after insertion of a temporary inlay). The tooth in question was disinfected by the application of 5 per cent tincture of iodine. After the removal of all carious dentine this disinfection was repeated. The roof of the pulp chamber and the coronal pulp were then removed with sterile instruments; they were flushed repeatedly with sterile saline. For initial cultures, a sterile paper point was brought in contact with the contents of the canal. For initial cultures from cases of old endodontic treatments, periapical secretion was collected and dentine particles were filed from the canal wall. After mechanical preparation, some dentinal material was filed from the wall of the dry canal and a paper point was introduced, care being taken to make eontact with the periapex. The paper point was left in situ for three minutes, wet with sterile saline, and transferred to the culture bottle. After the application of drugs, the canal was washed with $2 \mathrm{ml}$. of sterile saline. It has been shown ${ }^{1}$ that this technique prevents growth inhibition of the used medicaments.

Most cultures were taken by advanced dental students; a number of cases were treated by experienced dentists on the staff of our dental school.

Bacteriologic Procedures.-Primary cultures were taken in screw-capped $5 \mathrm{ml}$. bottles filled with brain-heart infusion broth (Difeo) to which 0.05 per 
cent of agar had been added (B.H.I.) All cultures were incubated at $37^{\circ} \mathrm{C}$. As soon as turbidity was observed, a microseopic preparation was made and the fluid was subcultured on three (later four) solid media. One blood agar plate was incubated aerobically. One blood agar plate and a Rogosa medium (pH 6.8) were incubated anaerobically. A Chapman plate, incubated aerobically, was added at a later period. In many cases these plates showed pure cultures. From the colony form and stained slides, a rough classification could be made. This was then confirmed, depending on the ease, by short sets of biochemical tests or by serologic tests. From mixed infections, different colonies were picked and subcultured for further identification.

Identification was, of necessity, preliminary. Important strains were kept for further study. With growing experience, it became possible (even with simple means) to allot each isolated strain to a definite group.

It is recognized that in the fluid primary culture some organisms might be selected and others suppressed. To arrive at more quantitative results, we plated the paper points directly and in another series triturated the paper points in broth with sterile sand and plated the resulting fluids. As neither of these experiments was encouraging, we adhered to the technique described.

The properties used for identification of the isolated strains will be deseribed with each organism. Serologic grouping of streptococei was accomplished by Fuller extraction and precipitation in eapillary tubes with commercial sera.

During a certain period of the study the value of two different culture media for primary culture was studied by taking duplicate cultures from each eanal. It has been shown before ${ }^{1}$ that duplicate cultures very often give equal results and that there is only slightly more chance for a positive culture from the first point than from the second. One paper point was cultured in brainheart infusion, the other in brain-heart infusion to which 0.1 per cent of glucose was added. (The commercial product contains a very small amount of glucose.) The order of these two cultures was alternated. The results are given in Fig. 1.

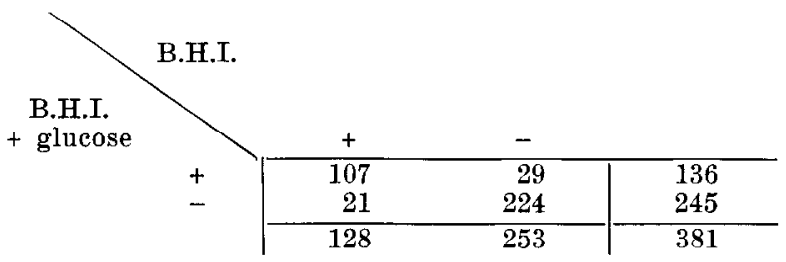

Fig. 1.

There seems to be a slight tendency toward B.H.I. plus glucose, which for this small number, of course, is not significant. From the 107 doubly positive cultures, the eultures with glucose showed growth at an earlier time in ten cases. The reverse was true in six cases. There was no obvious difference between the species of organisms cultured on the two media. 
Since January, 1958 , all primary cultures were made in B.H.I. plus glucose. A definite increase in the frequeney of lactobacilli from 2 to 8 per eent was observed after that time. There was possibly also a slight increase in Streptococcus salivarius but this may have been due to chance. The increased frequency of lactobacilli is obviously due to the improved growth conditions for this organism in the medium with glucose.

\section{RESUI,TS}

\section{Survey of Flora From All Cultures.-}

A total of 4,186 cultures were taken. Of these, 2,989 cultures (72 per cent) were negative and 1,141 (27 per cent) were positive. The frequency distribution of the isolated organisms is given in Table $I$ and Fig. 2. For details, the footnote to Table I should be consulted. In 903 cases one organism was isolated, whereas 238 cultures (21 per cent) contained more than one organism (mixed infections).

Tabie T. Strains Isolated From 4,186 Root Canat, Cultures

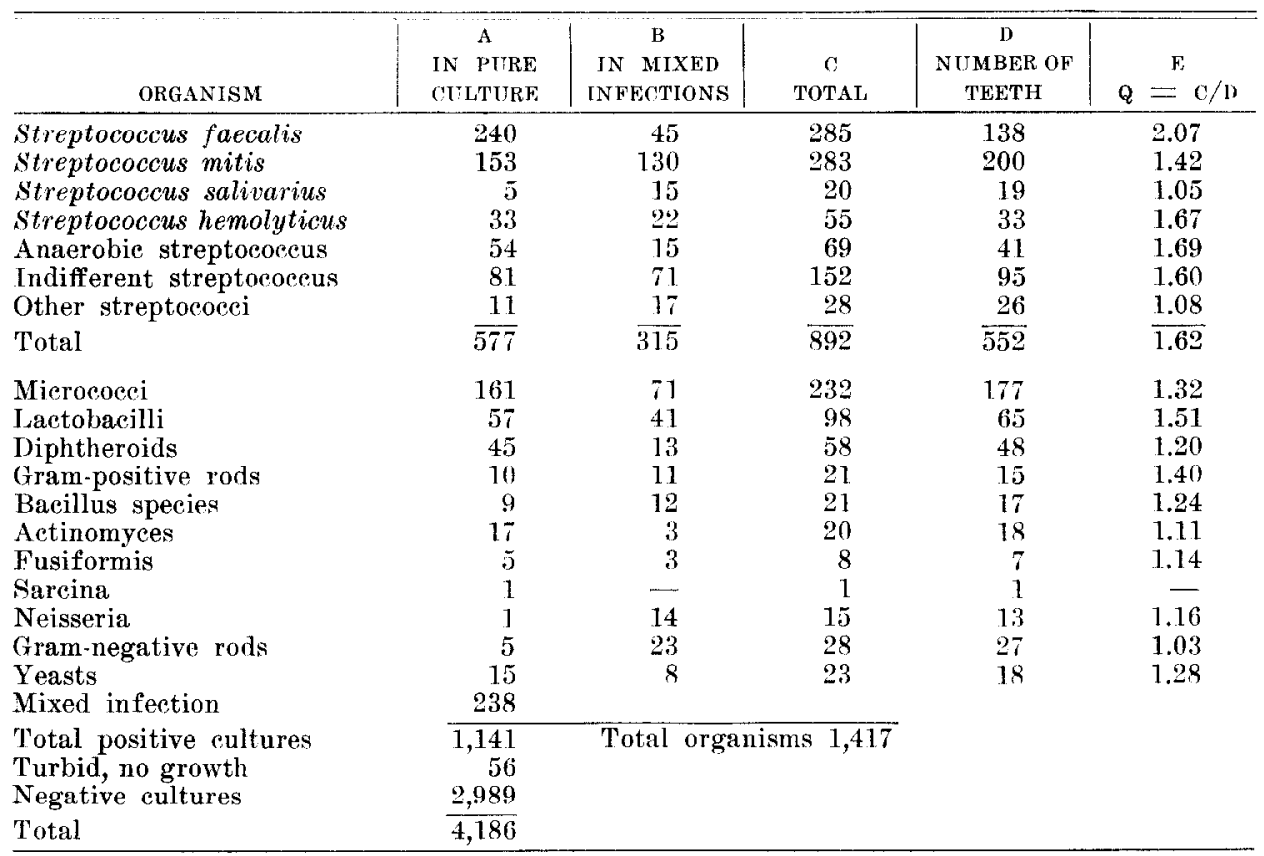

In fifty-six cases (1.5 per cent of all cultures) a slight turbidity, which could not be subcultured, was observed.

In column $B$ the trequency of any organism in mixed infections is given. As each mixea infection contributes at least two organisms, the total number of organisms (sum of column C) is appreciably higher than the total number of positive cultures.

Column D gives the number of teeth from which the strains of each group (column C) were isolated. The last column gives the total number of each organism divided by the number of teeth from which they were isolated. This ratio is a measure of the number of times the same organism was isolated from one canal. Organisms with a high ratio seem able to the same organism was isolated from one canal. Organisms with a high ratio seem able to
persist longer in the canal. (The ratio was not corrected for the number of teeth with more than one canal.) 
The preponderance of streptococei (51 per cent of pure cultures, 63 per cent of all organisms) is obvious. Micrococei, with 14 per cent, and lactobacilli, with 8.5 per cent, are next in number. Neisseria and Streptococcus salivarius, though abundant in the mouth, are rare and occur mainly in mixed infections ('Table I, column 2), which probably means that they cannot maintain themselves in the root canal. For the same reason, gram-negative rods are considered secondary invaders.

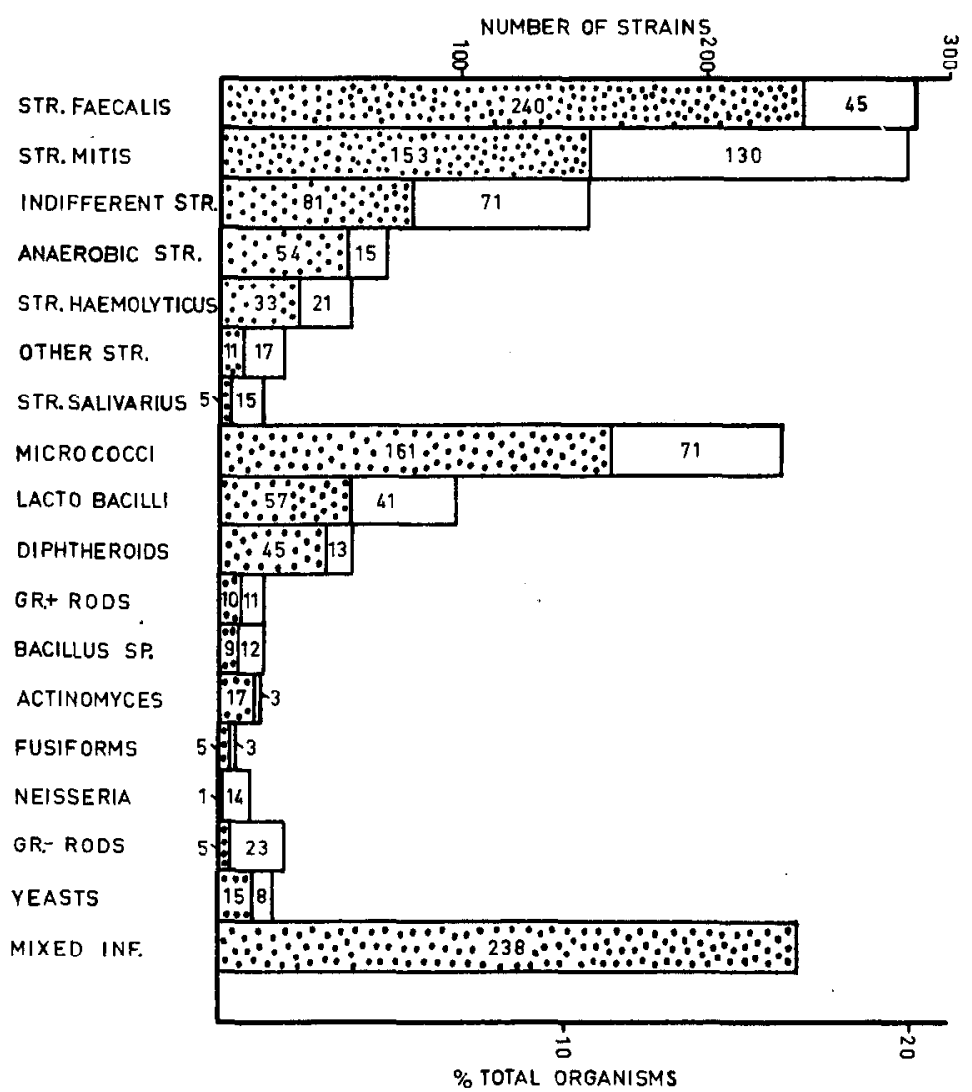

Fig. 2.-Frequency distribution of strains in 1,141 positive cultures. Shaded columns represent strains in pure culture; white columns represent additional strains from mixed infections. The per cent scale a.t the right refers to all organisms (shaded and white columns together). The shaded columns cannot be referred to this scale (see Table I).

Streptococcus faecalis: All strains presented under this heading showed characteristic large $\alpha$-hemolytic colonies. All strains withstood heating for 30 minutes at $60^{\circ} \mathrm{C}$; they fermented mannitol and esculin. Most strains were slightly more bile sensitive than the usual fecal strains. ${ }^{13}$ Many strains were grouped serologically; they were always group D.

The strains mentioned as Streptococcus faecalis in the table include Streptococcus liquefaciens. During a certain period of the study, all strains were classified in this respect. Thus, from July, 1957, to July, 1958, 158 strains of 
D) streptococei were cultured (144 pure cultures. 14 from mixed infectionsi. from which fifty nine liquefied golatin (37 per aont). Streptococas faeculis was often isolated more than once from one canal (mean ratio, 1.89). For Streptococcus liquefaciens, this ratio was 2.96 , which means that streptococcus liquefaciens persists much longer in root canals than any other organism.

Streptococcus mitis: All $\alpha$-hemolytic strains that could not otherwise be classified are presented under this heading. None of them produced slime from saccharose on solid medium. All strains that were tested serologically proved ungroupable; some strains were tested with Optochin and proved insensitive. During a certain period, a subdivision by colony form and fermentation reactions was attempted but appeared useless. Twenty randomly selected strains were tested for collagenase by incubating with Azocol ${ }^{12}$; only one strain was positive.

Streptococcus hemolyticus: From the fifty-five strains, forty-nine were grouped serologically. Twenty-eight (57 per cent) were group $\mathrm{F}$, fifteen (30 per cent) were group ( $\mathrm{t}$, two were group (, and four were not groupable with the available sera. Groups $\mathbf{F}$ and $\mathrm{G}$ were often isolated more than once from one patient (ratio about 2). For group $\mathrm{C}$ and the nongroupable strains, this ratio was 1 .

Indifferent streptococci: 'These strains formed minute colonies on blood agar and did not show any change in the blood medium. All strains wore insensitive to Optochin. They generally grew better anaerobically, and consequently the group merged with the next. In the beginning of the study, no classification was attempted. Later, the strains were tested serologically after Fuller extraction. From a series of torty strains, twenty-three reacted with the available sera: group F, ten; group C, six; group C, four; group D, two; and group E, one. Guthof ${ }^{10}$ and Wensinck ${ }^{19}$ deseribed analogous observations. All groupable strains fermented glucose, lactose, saccharose, maltose, salicin, and trehalose but not raffinose, arabinose, mannitol, sorbitol, or amylum. Lactose fermentation occasionally was retarded. Five of the ungroupable strains had the same fermentation pattern. The other ungroupable strains showed slightly different patterns. These facts point to a relationship between the groupable indifferent strains and hemolytic strains with the same cell-wall antigen. Ten indifferent strains were subcultured daily for several weeks in blood broth; none becamo hemolytic. The strains are under study and will be reported upon later. A relationship between these indifferent strains and hemolytic streptococei of groups $\mathrm{C}, \mathrm{G}$, and $\mathrm{F}$ might be important with respect to problems of focal infection and endocarditis.

Indifferent anaerobic streptococci: None of these strains showed $\alpha$ or $\beta$ hemolysis; they were completely indifferent toward blood. Judged by the (limited) fermentation tests described by Weinberg and associates, ${ }^{18}$ many of these strains could be described as streptococcus micros and some others seemed to belong to streptococcus intermedius. A large number of these strains were groupable. Of a series of ten strains, seven belonged to group $\mathrm{C}$. 
The fermentation patterns of these group C streptococci were equal to those of the former group, although three of the anacrobic strains did not ferment trehalose.

Although the evidence is incomplete, there seem to be many indications that the hemolytic, indifferent, and indifferent anaerobic streptococci isolated here are in some way related. That there exist other reasons to consider them as one group will be shown below.

Other streptococci: All the strains mentioned under this heading in Table I were $\alpha$ hemolytic. Some differed in colony form from the usual Streptococcus mitis. Some strains were scored as Streptococcus mitis and lost before further study; others remained completely unidentified. The whole group should probably be added to the mitis eategory.

Lactobacilli: Lactobacilli were rare (2 per cent of all organisms) during the first part of the investigation. Following the introduction of brain-heart infusion with glucose as primary culture medium, their number increased (8 per cent). Judging from the colony form on Rogosa medium, the strains were mainly Lactobacillus casei (or plantarum). Twelve strains were further identified. Of those, nine were Lactobacillus casei, one was $L$. fermenti, and one was $L$. plantarum.

Mixed infections: Of 196 mixed infections, 173 contained two organisms, and 20 contained three organisms; four organisms were cultured twice, and once five organisms were cultured. Among the double combinations Str. faecalis was found thirty-one times, Str. mitis 100 times, indifferent streptococci twenty-three times, micrococei eight times, and Str. hemolyticus ten times. Among the triple combinations, Str. faecalis was present eight times, and Str. mitis was present ten times.

Micrococci: Twice, a coagulase positive Micrococcus pyogenes was isolated. All other isolated strains showed white colonies on blood agar. All were anhemolytic and coagulase-negative. It will be argued below that the bulk of these strains are due to chance contaminations.

\section{Bacterial Flora From Different Clinical Situations.-}

There were complete case histories from 902 patients (canals). These involved 3,312 cultures, of which 993 (30 per cent) were positive. A slight turbidity in the primary culture which could not be subcultured (T.N.G. $=$ turbid, no growth)* was present in thirty-six cases. There were 2,317 (70 per cent) negative cultures.

The complete data, differentiated for vital pulps, open or closed necrotic pulps, and old endodontic cases, are given in Table IIA for initial cultures and in Table IIB for later cultures. Some details are discussed in the footnote to these tables. In Table III a summary is given, and it is convenient to discuss this table only. 


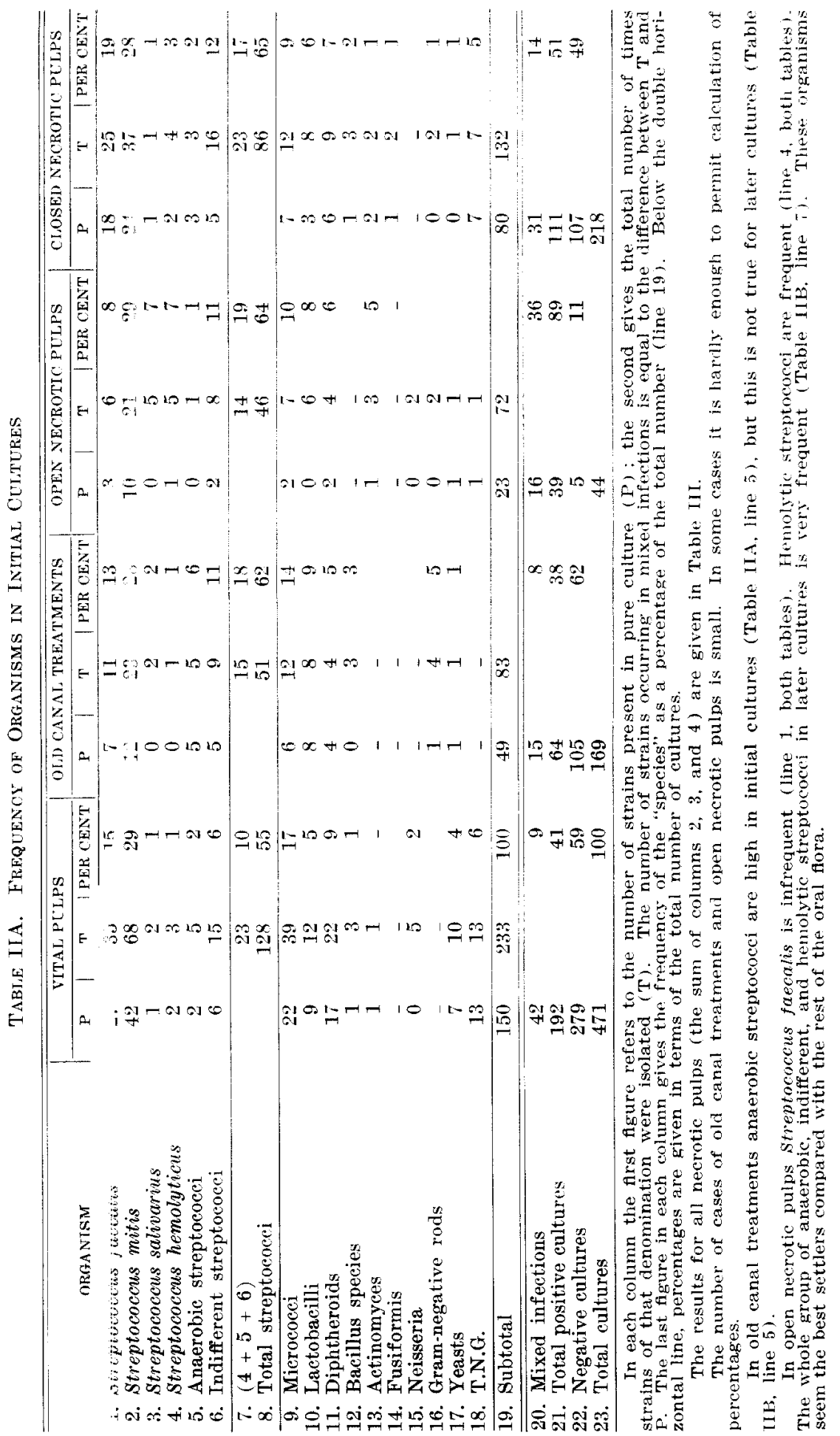




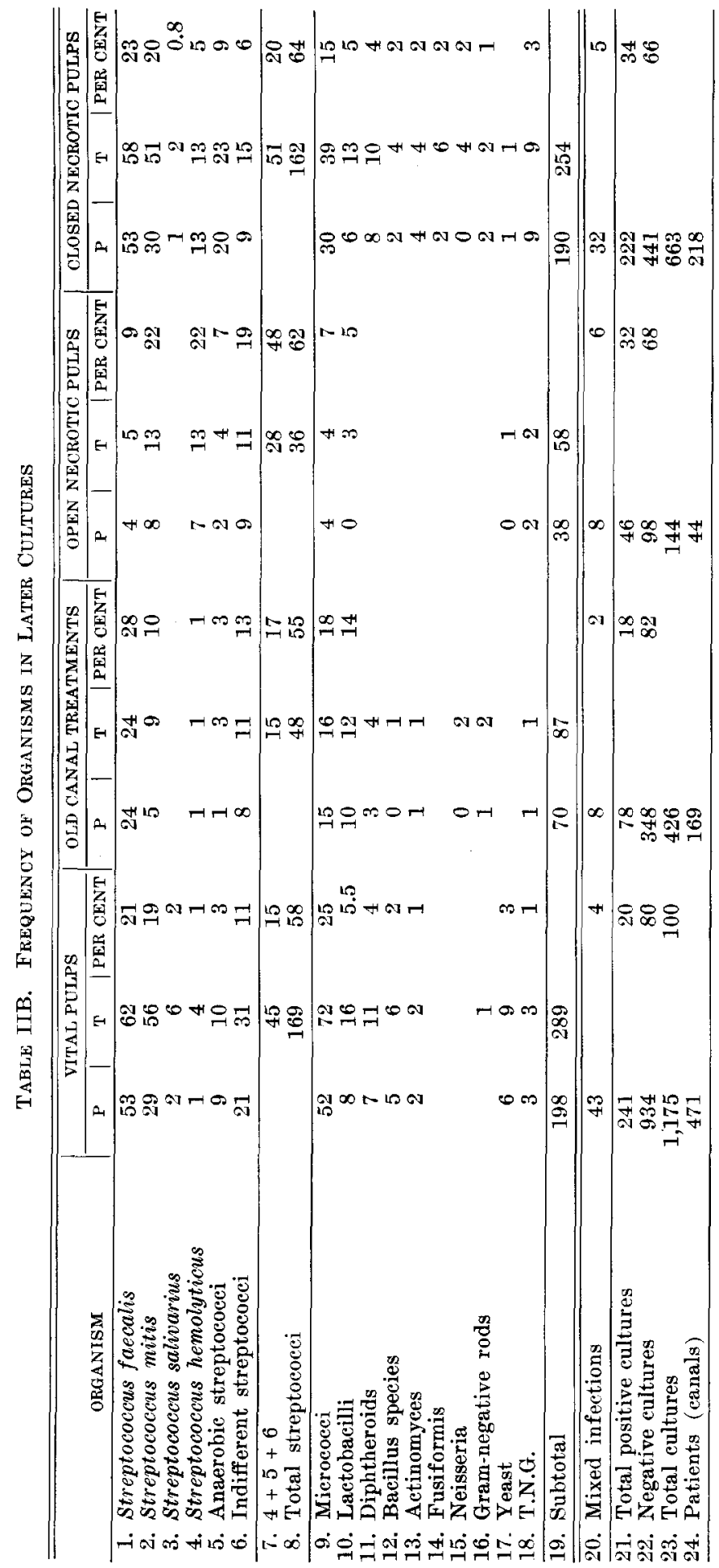




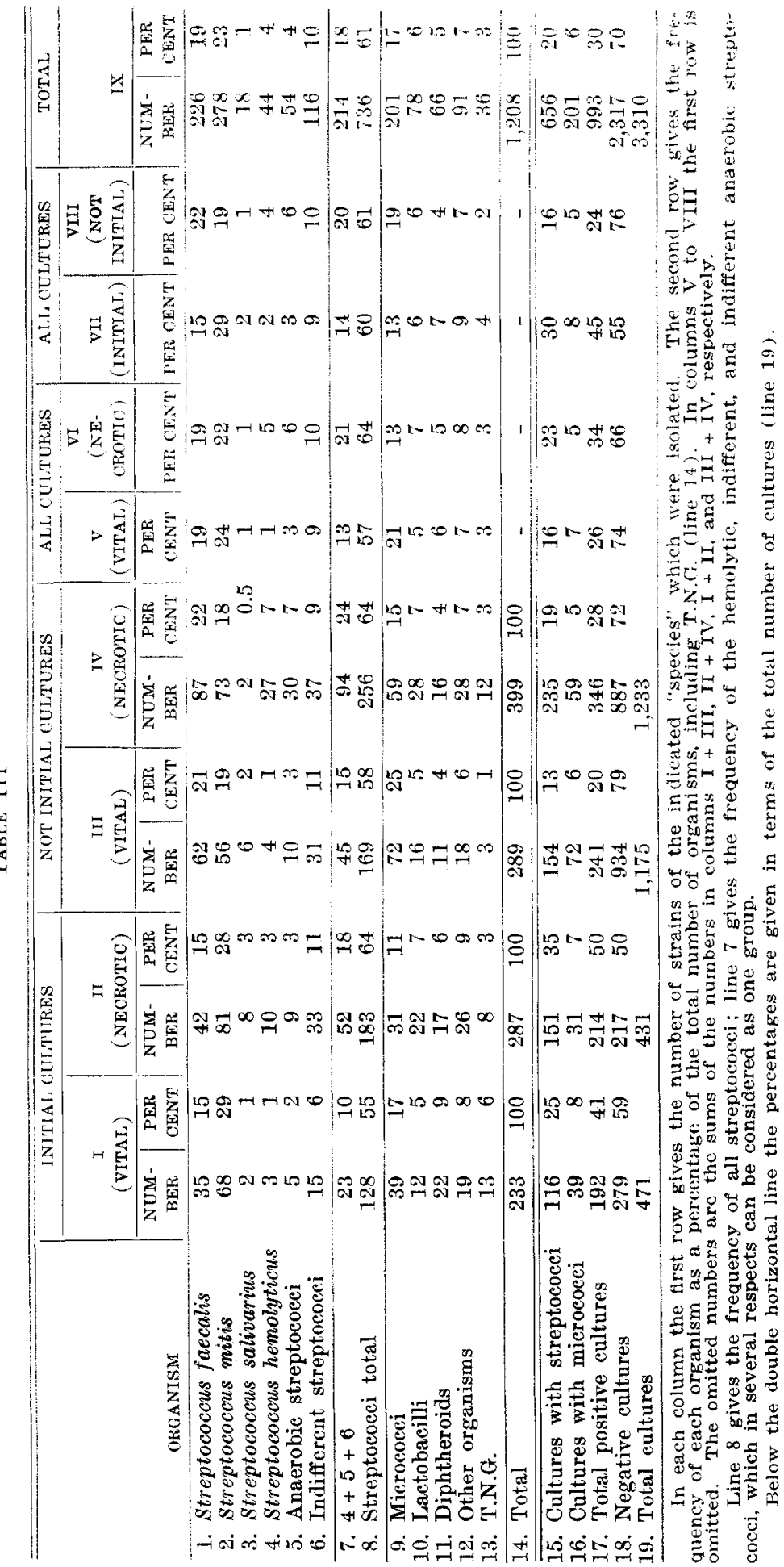


The last column (IX) lists the frequeney of each organism as percentage of the total number of organisms isolated. Streptococcus faecalis was present in 19 per cent, Streptococcus mitis in 23 per cent, and micrococei were present in 17 per cent. There is again a definite preponderance of streptococei, which form 61 per cent of all organisms (line 8) and occur in 20 per cent of all cultures (line 15).

Comparing initial cultures with later cultures, we find that the percentage of positive cultures is much higher among the initial cultures (45 per cent, column VII, line 17) than among later cultures (24.3 per cent, column VIII). As the cultures were taken at different stages of preparation and from all canals until one negative culture had been obtained (including one control culture immediately before filling which generally was negative ${ }^{1}$ ), this preponderance of negatives among later cultures is obvious.

If one is to understand the meaning of the frequency distribution of different organisms in the cultures, some theoretical considerations seem necessary.

The origin of the cultured bacteria is obviously of different kinds:

1. If we consider the procedure for taking initial cultures from accidentally exposed sound pulps, it is obvious that even with the strictest technique the chances of carrying bacteria of the carious eavity into the pulp during perforation of the roof of the pulp chamber are large. Contamination with salivary organisms through leaky rubber-dams should also be considered. This means that the number of positive eultures from pulps that did not contain bacteria before preparation will be large. In these cases the number of bacteria will probably be relatively small. As for the kind of organisms one may expect, all organisms that can be present in the deep earious lesion. If the mechanical preparation of the root canal does not immediately follow exposure of the pulp, some of these organisms might establish a clinical infection.

2. In the case of actual infection of the root canal, as in pulpitis and most cases involving necrotic pulps, the causal organism will nearly always have penetrated into the pulp from the carious lesion. Not all organisms will be able to maintain themselves in the pulp; only a small fraction of the caries flora will be able to overcome the natural defense of the living pulp. In infected and in necrotic pulps one may consequently expect a much smaller number of species, but the species that establish themselves will be present in relatively large numbers (unless the lesion has "sterilized" itself).

3. Finally, the ehance contamination of the culture from the air, from instruments, or from the hands of the operator indirectly is to be considered.

Differences Between Flora From Vital and Necrotic Pulps.-From the difference between the flora from vital and necrotic pulps, it would seem easy to 
decide which organisms were possible pathogens and which were chance corrtaminants. The difficulty is that the chance antamination during the opening of the canal is superimposed on the flora of the infected canal. Only quantitative evaluation of the number of hacteria of different species in the sample would permit a distinction betweon the cases mentioned under 1 and 2 above. With the present technique, any chance contaminant will grow in the fluid primary culture and confuse the issue.

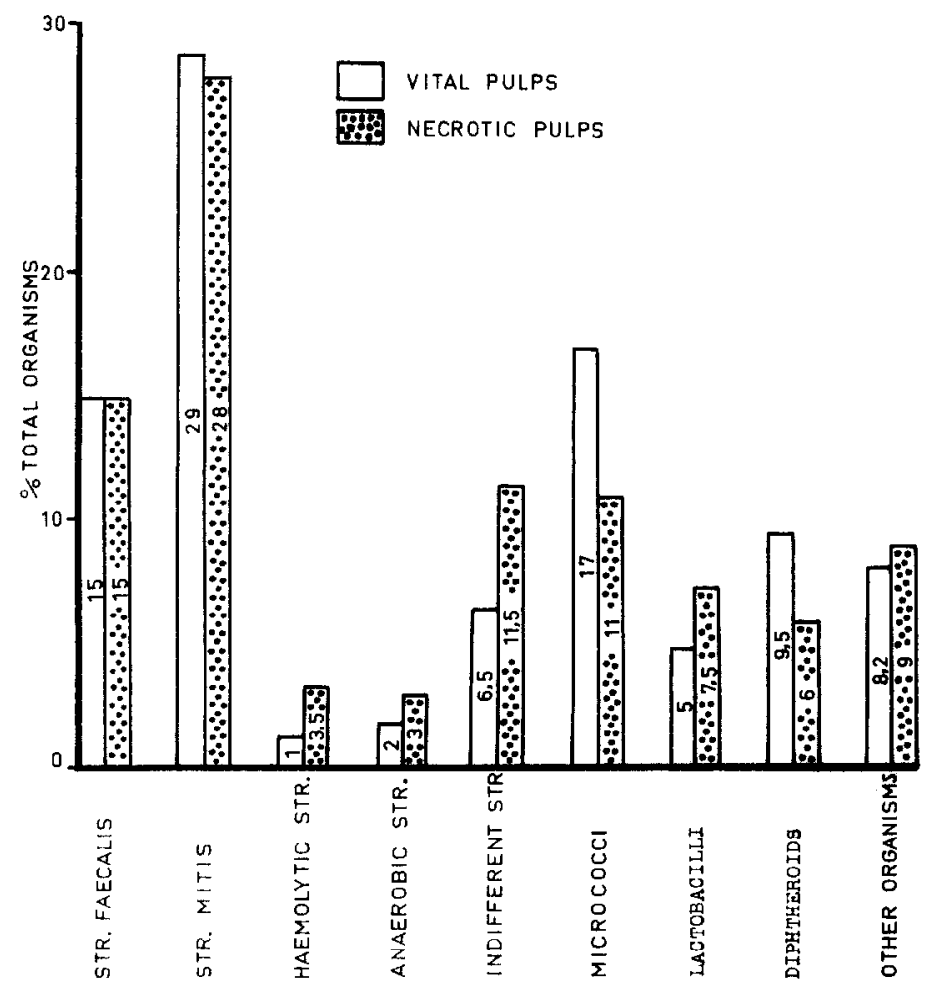

Fig. 3.-Frequency distribution of strains in initial cultures. The hemolytic, anaerobic. and indifferent streptococci and the lactobacilli occur with increased frequency in necrotic pulps. All other organisms are less frequent in necrotic than in vital pulps or show equal frequencies.

Nevertheless, sonte indications concerning an eventual pathogenic significance of some species might be obtained by comparing cultures from exposed sound pulps with those from necrotic pulps (Table III). On comparison of columns $I$ and II, III and IV, and $V$ and VT (line 17), it can be seen that the percentage of positive cultures from neerotic pulps is higher (41 to 50 per cent for initial cultures, $p<0.05$, for the other differences $p<0.01$ ). No large differences in the flora seem to exist. However, there is a definite increase in the percentage of streptococei (line 8), mainly due to the groups of hemolytic, anaerobic, and indifferent streptococei (Table III, line 7; 10 to 18 per cent for initial, 15 to 24 per cent for later, and 13 to 21 per cent for all cultures, all 
$p<0.05$; compare also Figs. 3 and 4 ). This seems to point to a possible role for these organisms as primary invaders. Streptococcus mitis is equally frequent in all eases.

The group of micrococei appears to be found in a fairly constant percentage of all eultures in all groups (Table III, line 16; only the difference between columns VII and VIII has a $\mathrm{p}<0.05$ ). If calculated as percentage of all organisms (line 9), the micrococei are less frequent in cultures from necrotic pulps $(21 \rightarrow 13$ per cent columns $\mathrm{V}$ and VI; $\mathrm{p}<0.01$ ). This is obviously due to the higher percentage of positive cultures from necrotic pulps. They are also less frequent in initial cultures than in later cultures (line 9, columns VII and VIII). This, again, is due to the higher frequeney of negatives among the

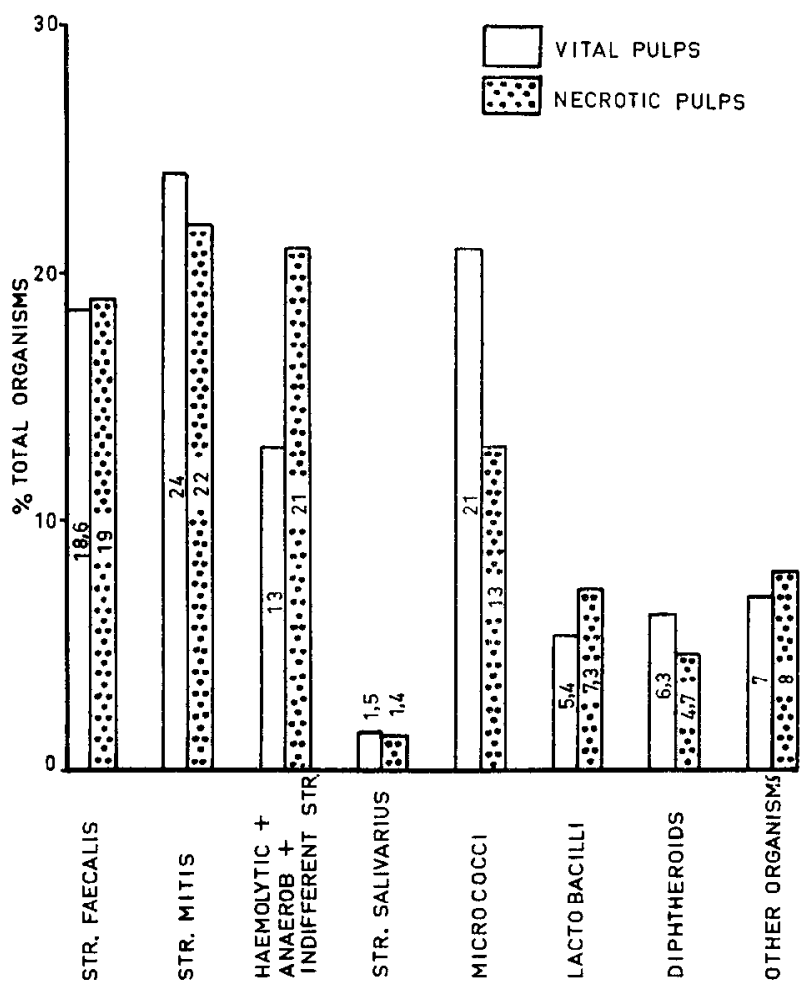

Fig. 4.-Frequency distribution of strains in all cultures. The group of hemolytic, anaerobic, and indifferent streptococci occurs with increased frequency in cultures from necrotic pulps.

later cultures. This seems to indicate that they are mainly chance contaminants. This view is supported by the fact that only coagulase-negative micrococei (abundant on human skin) were found. The point is further stressed by the much lower percentage of staphylocoeci found in cultures taken by experienced dentists than in those taken by students (Table IV).

Organisms Persisting in the Root Canal.-In comparing the flora of initial cultures with that of later cultures, one might acquire some information about 
Tabie 1V. Frequency of Micrococel in Cultures From Experienced Dentists ani STUDENTS

\begin{tabular}{|c|c|c|c|c|}
\hline \multirow[b]{2}{*}{ OUITURE } & \multicolumn{2}{|c|}{ DENTIS'TS } & \multicolumn{2}{|c|}{ STUDENTS } \\
\hline & NUMBER & PER CENT & NUMBER & PER CENT \\
\hline Cultures with microcoeci & 17 & 2.5 & 184 & 7 \\
\hline Cultures with streptococei & 133 & 20 & 523 & 20 \\
\hline Cultures with other bacteria & 26 & 4 & 144 & 5.4 \\
\hline Positive cultures & 172 & 26 & 821 & 31 \\
\hline Negative eultures & 493 & 74 & 1,824 & 69 \\
\hline Total & 665 & & 2,645 & \\
\hline Micrococci (per cent from positive cultures) & & 10 & & 22 \\
\hline
\end{tabular}

The sum of the cultures with micrococci, streptococci, and other bacteria is not equal to the number of positive cultures, as there were four mixed infections with micrococci and streptococci in the dentist group and twenty in the student group.

The most experienced dentist had 1.8 per cent cultures with micrococei ; the other 3.5 per cent.

the organisms that are able to persist in the root canal. On comparison of columns I and III, II and IV, and VII and VIII (Table III), there seem to be no large differences. This is due again to the large background of chance contaminations. Studied more closely, however, it appears that Streptococcus faecalis is present more frequently in later cultures (15 to 22 per cent, line 1 , columns VII and VIII). The same is true for hemolytic, anaerobic, and indifferent streptococei $(14 \rightarrow 20$ per cent, line 7$)$. Streptococcus mitis is less

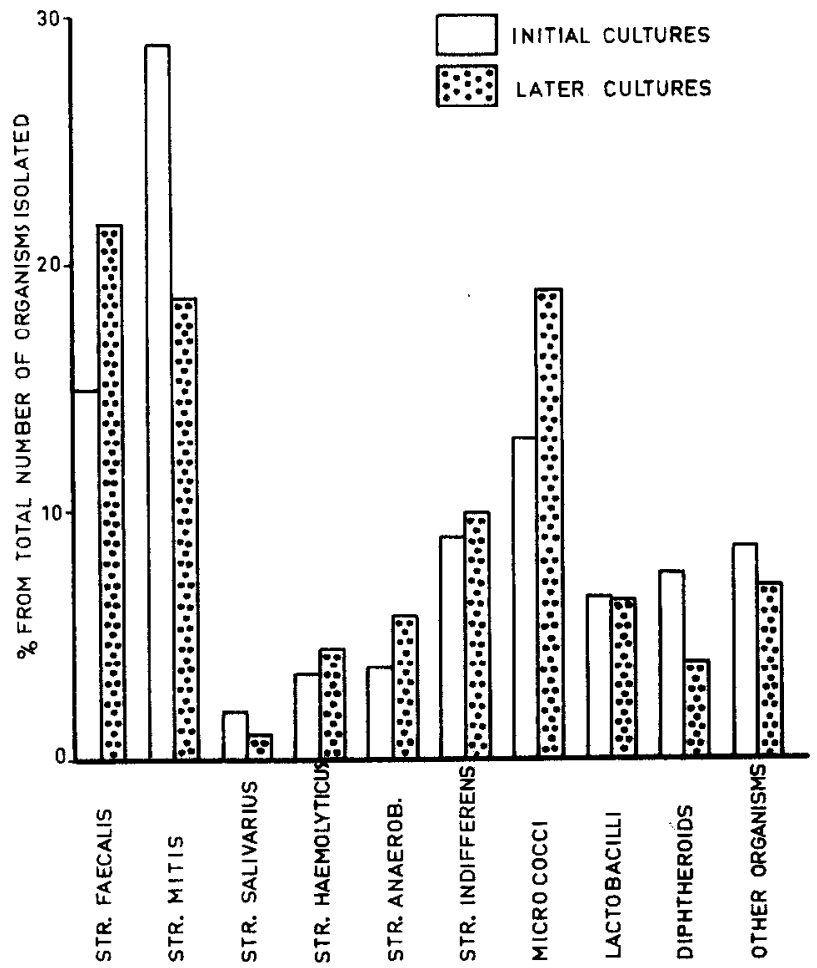

Fig. 5.-Frequency distribution of strains in all cultures. Organisms that persist in the root canal will be present with higher frequency in later cultures. These are streptococous faecalis, the hemolytic, anaerobic, and indifferent streptococci. The apparent increase of micrococci is due to other causes (see text). 
frequent in later cultures. Streptococcus faecalis, Streptococcus hemolyticus, and the anaerobic streptococci seem to persist better in the root canal than do other organisms (Fig. 5).

Another approach to the possible role of the isolated strains in root canal infection is the study of cases in which the same organism was isolated more than once from the same canal. This was performed by counting the cases in which the organism found in the first culture was again found (consccutively) in the second, third, etc., cultures from the same canal. In the cultures from vital pulps there were forty-three ( 24 per cent) such cases where an organism was found more than once. In the group from necrotic pulps this number was eighty (39 per cent). The distribution of organisms is given in Table $V$.

In some cases the numbers seem small. It should be remembered, however, that the probability for a consecutive series of three cultures of, for example, Streptococcus hemolyticus is extremely small (compare note under Table V). It is obvious that some organisms persist whereas others do not ('lable $V$, last three columns). The organisms that persist longest (that is, maintain themselves best) seem to be Streptococcus hemolyticus, Streptococcus faecalis, indifferent streptococei, indifferent anaerobic streptococci, and perhaps lactobacilli and Streptococcus mitis. All other organisms are present only in the initial cultures, and as they disappear after preparation they do not seem to have settled themselves in the dentine or in the periapical tissues. The separate results for vital and necrotic pulps are given in the first half of Table V. Long series of consecutive cultures occur mainly in the group with necrotic pulps. For Streptococcus faecalis, Streptococcus hemolyticus, the indifferent streptococei, and lactobacilli, the percentage of consecutive positives is higher in the cultures from necrotic pulps. This is not so for micrococei and Streptococcus mitis; consequently, we consider these organisms as less important.

In many cases given as consecutive series of cultures of Streptococcus faecalis, it was definitely Streptococcus liquefaciens which was isolated. If we divide the number of times Streptococcus liquefaciens was isolated by the number of all canals in which Streptococcus liquefaciens was found, the ratio is 3 , as has already been discussed. As the ratio for Streptococcus faecalis is only 2, there is no doubt that Streptococcus liquefaciens persists better in the root canal than Streptococcus faecalis.

The fact that long series of consecutive cultures positive for one organism can occasionally be obtained from vital pulps requires explanation. Apart from the obvious difficulty in clinical diagnosis, we think that in some of these cases a contamination during opening of the canal could have established itself as a clinical infection in the period between instrumental entry and mechanical preparation (generally one week).

In an analogous calculation the cases with more than one culture with the same organism were counted but the requirement of consecutiveness was omitted. The results pointed in the same direction. The number of cases was somewhat larger. The order of the organisms, in terms of frequency of three or more 


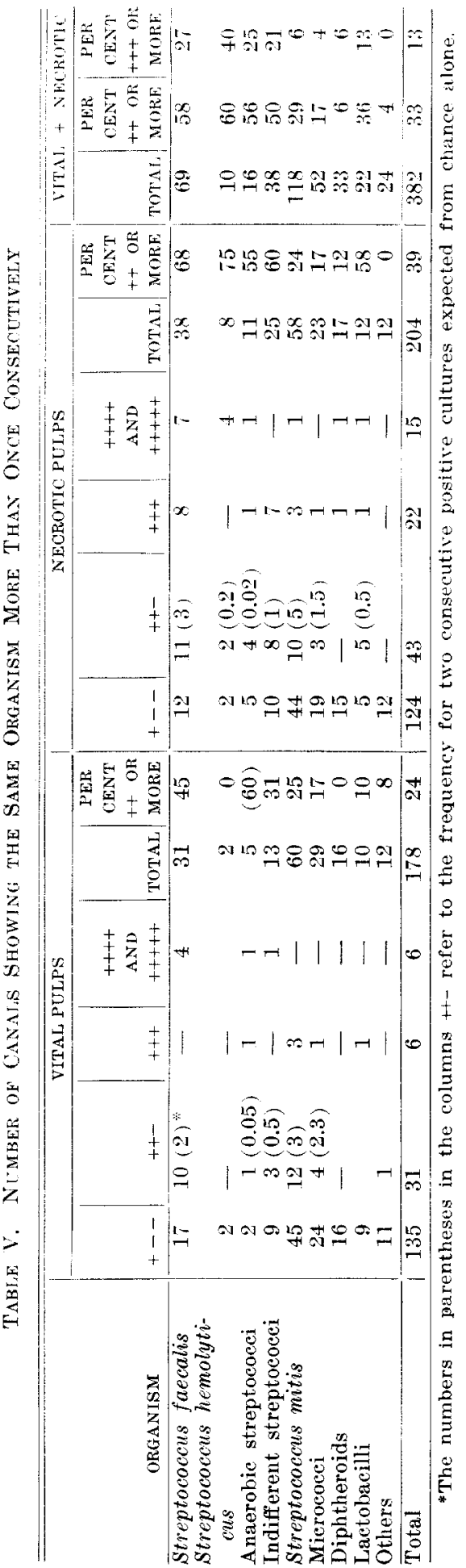


positive cultures from one patient, was again Streptococcus faecalis, Streptococcus hemolyticus, anaerobic streptococci, indifferent streptococci, lactobacilli, Streptococcus mitis and micrococci.

\section{DISCUSSION}

The chance of carrying bacteria from the carious cavity into the root canal during the opening of the canal is relatively great. Consequently, a large fraction of root canal cultures, even those from exposed sound pulps, will be positive due to this type of contamination. The large background of chance contamination hampers the analysis of results. This large background is due partly to the fact that most cultures discussed in this article were taken by relatively inexperienced operators. The study of an cqually large amount of material gathered by experienced dentists would be much more useful.

Another serious difficulty in the interpretation of results is the fact that the primary culture had to be made in fluid medium, in which some organisms will have been selected and others suppressed. Development of a method for a more quantitative evaluation of the root canal flora is obviously of great importance. Until such a method has been developed, it will be difficult to arrive at definite conclusions concerning the pathogenic importance of the isolated organisms.

Recognizing these limitations, however, we believe that an analysis of the available results permits some tentative conclusions.

The coagulase-negative micrococci, which form 17 per cent of all organisms, are to be considered as chance contaminants. Irrespective of the conditions of the canal or the stage of treatment, they occur with equal frequency in all cultures. Expressed as a fraction of total organisms, they are less frequent in infected pulps than in sound pulps. The number of two consecutive cultures with micrococei from one canal does not differ greatly from the number expected from chance alone (Table V). Furthermore, there is a definite negative correlation between the frequency of micrococci and the technical skill of the operator (Table IV).

For Streptococcus mitis the data are much less definite. This organism might also be a chance contaminant, as it occurs with equal frequency in cultures from vital and from necrotic pulps and is less frequent in later cultures (Table III). The number of consecutive cultures with Streptococcus mitis from one canal is equal for vital and for necrotic pulps (Table VI) and, although it is larger than from chance alone, the difference is much less than for any other organism (cxcept micrococi). As the group called Streptococcus mitis is probably heterogeneous, it is quite possible that it still contains a fraction of possible pathogens. A large fraction are probably chance contaminants, however, as was to be expected from the organism's abundance in the mouth.

Among the other streptococei, the hemolytic indifferent and anaerobic indifferent streptococci seem to be serious candidates for a possible role as the cause of pulp infections. The same seems to be true for the group D streptococci, although the evidence is less unequivocal here. 
The hemolytic, indifferent, and anderobic indifferent streptococei can be treated as one group because, irrespective of the differences in oxygen requirement and hemolysis, they show relationships in cell-wall antigens and fermentation patterns. In the future, it would be better perhaps to give priority to the serologic character and discuss the significance of $\mathrm{F}, \mathrm{G}$, and $\mathrm{C}$ streptococe separately. At the moment, we will take the whole group together.

As they are more frequent in cultures from necrotic pulps than from vital pulps (Figs. 2 and 3 ), more frequent in later than in first cultures, have a high mean ratio of cultures per canal (Table I), and occur more often in consecutive series of positive cultures from one canal, especially in necrotic pulps ( Table V), these streptococci can be considered as possible pathogens. The fact that nonhemolytic streptococei seem to act as possible pathogens might be of importance from the point of view of focal infection and endocarditis.

The $\mathrm{D}$ streptococei persist tenaciously in root canals, as can be seen from their higher frequency in later cultures (Table III), from the high ratio of organisms per canal (Table I), and from the long series of consecutive cultures from one canal (Table $V$ ). Streptococcus liquefaciens is even more persistent in this respect than Streptococcus faccalis. D streptococei are difficult to eliminate from the root canal.

This persistence seems to point to a possible role as a pathogen. One would then expect a higher frequency in cultures from infected pulps than from vital pulps. The observed difference, however, is negligible (Fig. 4) or absent (Fig. 3). It should be remembered that the frequency of chance contaminants (for example, micrococci and diphtheroids) is definitely lower in cultures from necrotic pulps (Fig. 4). The D streptococci take an intermediate position between the chance contaminants which occur less frequently in cultures from necrotic pulps and the organisms which are considered possible pathogens because they are more frequent in cultures from necrotic pulps. We think that the expected higher frequency of $D$ streptococei in cultures from necrotic pulp is obscured by the large chance of contamination by this organism. The high frequency of the number of series of consecutive positive cultures of $D$ streptococci from necrotic pulps (68 per cent) compared with the frequency from vital pulps (45 per cent, Table V) supports the view that D streptococei are possible pathogens.

These results seem to point to a large chance of contamination with D streptococci. In some cases they seem to be able to cause clinical infections, and if they do they are difficult to eliminate. For these reasons, we think that they should be considered potential pathogens.

Lactobacilli seem also to be more frequent in necrotic pulps and are occasionally able to persist (Table $V$ ). Without further data, no conclusion is warranted sinee the numbers are too small.

\section{SUMMARY}

The bacteriologic results from more than 4,000 root canal cultures are discussed. Streptococci form 61 per cent of the isolated organisms. Among these, 
the group of hemolytic, indifferent, and indifferent anaerobic streptococci, mainly belonging to serologic groups $\mathrm{F}, \mathrm{G}$, and $\mathrm{C}$, seem the most serious possible pathogens. D streptococei (especially Streptococcus liquefaciens) are difficult to eliminate from the root eanal and are also potential pathogens. Streptococcus mitis seems of much less importance, and all other organisms are to be considered as chance contaminants.

Many persons from the staff of the Laboratory have helped with the isolation and identification of organisms or with the calculation of results. Special thanks are due Mr. R. Braakenburg v. Backum, Mr. H. Ottens, Miss H. G. S. Vreede, Miss W. I. A. v. Thienen, and Miss M. H. G. Tressel.

\section{REFERENCES}

1. Van Amerongen, J., Slaterus, W. J., and Eggnik, C. O.: De toepassing van bacteriologische controles in de endodontie. I. (English summary), Nederl. tijdschr. tandheelk.64: 494-520, 1957.

2. Van Amerongen, J.: The Bacteriological Control of Endodontic Treatment. II. The Significance of Two Consecutive Negative Cultures (English text), Nederl. tijdschr. tandheelk. 64: 572-577, 1957.

3. Van Amerongen, J., Eggink, C. O., and Klevant, F. J. N.: De toepassing van bacteriologische controles in de endodontie. IV. De invloed van mechanische preparatie en het appliceren van medicamenten op de infecties van het wortelkanaal en het peri-apicale gebied, Nederl, tijdschr. tandheelk. 65: 145-167, 1958.

4. Van Amerongen, J., Eggink, C. O., and Lamers, A. C.: The Bacteriological Control of Endodontic Treatment. III. Bacteriological Examination of Root Canals, Treated With Polyantibiotics and Other Medieaments, Nederl. tijdschr. tandheelk. 65: $65-76,1958$.

5. Appleton, J. L. T.: Bacterial Infection, ed. 4, Philadelphia, 1950, Lea \& Febiger.

6. Auerbach, M. B.: Antibiotics Versus Instrumentation in Endodonties, New York State Dent. J. 19: 225-229, 1953.

7. Buchbinder, M.: Results of Culturing Root Canals by a Simple Anaerobic Method, J. D. Res. 19: 426, 1940.

8. Buchbinder, M.: A Statistical Comparison of Cultured and Non-Cultured Root Canal Cases, J. D. Res. 20: 93-97, 1941.

9. Grossman, L. I.: Root Canal Therapy, ed. 4, Philadelphia, 1955, Lea \& Febiger.

10. Guthof, O.: ťber pathogene "vergrünende Streptokokken," Zentralbl. Bakt. Orig. 166: 553-565, 1956.

11. Hettche, O.: Die Prüfung des Wurzelkanals auf Sterilität, Deutsche Zahn. Ztschr. 7: 573-576, 1952 .

12. Lucas, R, B., and Thonard, .T. C.: The Action of Oral Bacteria on Collagen, .T. D. Res. 34: 118-123, 1955.

13. Morris, E. O.: The Bacteriology of the Oral Cavity. III. Streptococeus, Brit. Dent. J. 96: 95-108, 1954.

14. Morse, F. W., and Yates, M. F.: Follow-Up Studies of Root-Filled Teeth in Relation to Bacteriologic Findings, J. Am. Dent. A. 28: 956-972, 1941.

15. Ostrander, F. D., Crowley, M. C., and Dowson, J.: A Clinical Study of the Treatment of Root Canal and Periapical Infections With Penicillin, .J. D. Res. 26: 403-408, 1947.

16. Shay, D. E.: The Selection of a Suitable Medium for Culturing Root Canals, J. D. Res. 26: $327-333,1947$.

17. Stewart, G. G.: The Importance of Chemomechanical Preparation of the Root Canal, Oral Surg., Oral Med. \& Oral Path. 8: 993-998, 1955.

18. Weinberg, M. R., Nativelle, R., and Prévot, A. R.: Les microbes anaérobies, Paris, 1937, Masson \& Cie, p. 1009 .

19. Wensinck, F., and Renaud, H.: Murine Group G Streptococei, Brit. J. Exper. Path. 38: $489-493,1957$. 